treatment options. In contrast, those diagnosed since 2005 linked to care promptly and felt they received appropriate medical attention.

Retention in care - Across the generations, once linked to care participants were committed to attending appointments and taking medications. Occasional lapses were explained by external issues such as drug misuse or household disruption, rather than their relationship with the clinic. Some reported concern at the recently reduced frequency of appointments, and the increasing role of primary care.

Viral suppression among those on ART - Most participants on ART had undetectable viral load and good adherence. Actual or anticipated co-morbidities worried them more than HIV, however, wider discussions about NHS cost-cutting have raised patient anxiety about accessing the 'best' treatments.

Conclusion The high standard of UK's HIV treatment cascade reflects strong relationships between patients and staff, which service changes could undermine. Being sensitive to how patients experience different stages of decision-making and the wider influences on their behaviour is vital towards sustaining high retention along the cascade.

Disclosure of interest statement This study is funded by a grant from the Imperial NIHR BRC and the Imperial Healthcare Charity. No pharmaceutical grants were received in the development of this study.

\section{P17.21 FAILURE TO ENGAGE AS KEY FACTOR OF LOSS TO FOLLOW-UP FROM CARE AND TREATMENT AMONG HIV-INFECTED CHILDREN IN BOTSWANA: A CASE- CONTROL STUDY}

${ }^{1,2} \mathrm{EM}$ Machine*${ }^{*},{ }^{1} \mathrm{SL}$ Gillespie, ${ }^{2} \mathrm{~N}$ Homedes, ${ }^{2} \mathrm{~B}$ Selwyn, ${ }^{2} \mathrm{MW}$ Ross, ${ }^{1,3} \mathrm{G}$ Anabwani, ${ }^{1} \mathrm{G}$ Schutze, ${ }^{1} \mathrm{M}$ Kline. ${ }^{1}$ Baylor College of Medicine; ${ }^{2}$ University of Texas School of Public Health; ${ }^{3}$ Botswana-Baylor Children's Clinical Centre of Excellence

\subsection{6/sextrans-2015-052270.599}

Introduction Loss to follow-up (LTFU) is a critical factor in determining clinical outcome in HIV treatment programs. Identifying modifiable factors of LTFU is fundamental for designing effective patient retention interventions. We analysed factors contributing to children LTFU from a treatment program to identify those that can be modified.

Methods A case-control study involving 313 children was used to compare the sociodemographic and clinical characteristics of children LTFU (cases) with those remaining in care (controls) at a large paediatric HIV care setting in Botswana. We traced children through caregiver contacts and those we found, we conducted structured interviews with the patients' caregivers.

Results Children $<5$ years were twice as likely as older children to be LTFU $(20 \cdot 6 \%$ vs. $7 \cdot 8 \%$ and $79 \cdot 4 \%$ vs. $92 \cdot 2 \%$ respectively, $\mathrm{p}<0 \cdot 01)$. Approximately half $(47 \cdot 6 \%, \mathrm{n}=51)$ of LTFU patients failed to further engage in care after just one clinic visit, as compared to less than $1 \%(\mathrm{n}=2)$ in the control group $(\mathrm{p}<$ $0 \cdot 01$ ). Patients LTFU were more likely than controls to have advanced disease, greater immunosuppression, and not to be receiving Antiretroviral Therapy (ART). Among interviewed patient caregivers, psychosocial factors (e.g. stigma, religious beliefs, child rebellion, disclosure of HIV status) were characteristic of patients LTFU, but not of controls. Socioeconomic factors (e.g. lack of transportation, school-related activities, forgetting appointments) were cited predominantly by the controls.
Conclusion Paediatric patients and their caregivers need to be targeted and engaged at their initial clinic visit, with special attention to children $<5$ years. Possible interventions include providing psychosocial support for issues that deter patients from engaging with the clinic. Collaboration with communitybased organisations focused on reducing stigma may be useful in addressing these complex issues.

Disclosure of interest statement Funding for this study was made possible through Fogarty International Centre of the National Institutes of Health, (M. W. Kline - Principal Investigator) under grant number D43 TW01036.

\section{P17.22 PROVIDERS' PERCEPTIONS OF THE CAUSES OF LOSS TO FOLLOW-UP OF HIV-INFECTED CHILDREN IN BOTSWANA}

1,2EM Machine*, ${ }^{1} \mathrm{SL}$ Gillespie, ${ }^{2} \mathrm{~N}$ Homedes, ${ }^{2} \mathrm{MW}$ Ross, ${ }^{2} \mathrm{BJ}$ Selwyn, ${ }^{1,3} \mathrm{GM}$ Anabwani, ${ }^{1} \mathrm{MW}$ Kline. ${ }^{1}$ Baylor College of Medicine; ${ }^{2}$ University of Texas School of Public Health; ${ }^{3}$ Botswana-Baylor Children's Clinical Centre of Excellence

\subsection{6/sextrans-2015-052270.600}

Introduction Healthcare providers (nurses, physicians, and social workers), by virtue of their experiences in interacting with HIVinfected children and their caregivers, are an important source of information on the causes of loss to follow-up (LTFU). We explored perceptions of healthcare providers regarding factors that lead to paediatric HIV-infected patients becoming lost to follow-up from care and treatment.

Methods The study was conducted at a large paediatric HIV clinic in Gaborone, Botswana and involved conducting in-depth interviews with clinical staff $(n=10)$. The interviews targeted information about the magnitude of LTFU problems and possible solutions as perceived by the healthcare providers.

Results Respondents perceived factors of LTFU to include issues of HIV-related stigma, caregiver's religious beliefs of being healed, teenage-child rebellion, and concerns about disclosure of their HIV status to others, were characteristic of the patients LTFU. The results also revealed that mental health issues such as depression might not be adequately addressed in HIV clinic settings, perceived as a key underlying factor of LTFU.

Conclusion Our study underscores the psychosocial nature of the issues of LTFU and the need to develop a more holistic approach to treating HIV-infected children.

Disclosure of interest statement Funding for this study was made possible through Fogarty International Centre of the National Institutes of Health, (M. W. Kline - Principal Investigator) under grant number D43 TW01036.

\section{P17.23 IMPLEMENTING PRIORITISED HIV LINKAGE-TO-CARE AND CONTACT TRACING AMONG INDIVIDUALS WITH HIGH HIV VIRAL LOAD IN BALTIMORE, MARYLAND, USA: RESULTS FROM A PILOT PROGRAM}

\footnotetext{
${ }^{1,2} \mathrm{CM}$ Schumacher ${ }^{*},{ }^{2,3} \mathrm{M}$ Joe, ${ }^{2,3} \mathrm{C}$ Ramsey, ${ }^{1} \mathrm{~A}$ Greiner Safi, ${ }^{2,4,5} \mathrm{P}$ Chaulk, ${ }^{1,6} \mathrm{JM}$ Jennings. ${ }^{1}$ Center for Child and Community Health Research, Department of Pediatrics, Johns Hopkins University School of Medicine, Baltimore, Maryland, USA; ${ }^{2}$ Baltimore City Health Department, Baltimore, Maryland; ${ }^{3}$ Centers for Disease Control and Prevention, Atlanta, GA, USA; ${ }^{4}$ Division of Infectious Diseases, Department of Medicine, Johns Hopkins University School of Medicine, Baltimore, Maryland, USA; ${ }^{5}$ Department of Health Policy and Management, Johns Hopkins University Bloomberg School of Public Health, Baltimore, Maryland, USA; ${ }^{6}$ Department of Epidemiology, Johns Hopkins University Bloomberg School of Public Health, Baltimore, Maryland, USA
}

10.1136/sextrans-2015-052270.601 
Background Because of increased transmission potential, the US Centres for Disease Control and Prevention (CDC) recommends prioritising high HIV viral load ( $\geq 50,000$ copies/mL, HVL) individuals for routine follow-up services, including linkage-to-care and partner services. However, little guidance exists on operationalizing this recommendation. In June 2014, the Baltimore City Health Department developed and implemented a pilot program to prioritise HVL individuals for follow-up services. The objective of this analysis is to describe the pilot program and evaluate process outcomes for follow-up services pre- and post-pilot program implementation.

Methods This pilot program was modelled after a protocol for responding to congenital syphilis. Disease Intervention Specialists (DIS) were trained to locate, administer partner services interviews and link-to-care HVL individuals with increased urgency and effort compared to other HIV cases. A DIS supervisor reviewed each HVL case before closing to ensure adequate response and documentation. We used a pre-post design to evaluate the pilot and compared linkage-to-care and contact tracing outcomes for HVL individuals post pilot implementation (postpilot, June 2014-January 2015) to a similar time period prior to implementation (pre-pilot, June 2013-January 2014).

Results There were 23 pre-pilot and 17 post-pilot HVL cases (n $=40$ ). DIS were more likely to link HVL individuals to care (59\% pre-pilot vs. 65\% post-pilot), and complete partner services interviews (39\% pre-pilot vs. 59\% post-pilot). Among HVL individuals who completed interviews, DIS were more likely to obtain sex partner meeting place information (33\% pre-pilot vs. $40 \%$ post-pilot) and locating information for disclosed sex partners (16\% pre-pilot vs. $39 \%$ post-pilot) in the post-pilot period compared to the pre-pilot period.

Conclusion This pilot program demonstrates one successful method to operationalize CDC guidelines regarding prioritisation of HVL individuals. Future work will evaluate additional outcomes of this program, including HIV testing among sex partners and at sex partner meeting places disclosed by HVL individuals.

Disclosure of interest statement The authors have no conflicts of interest to disclose.

\section{P17.24 DELAYED LINKAGE TO CARE IN A THIRD OF HIV POSITIVE INDIVIDUALS IN THE NETHERLANDS: OPPORTUNITIES TO IMPROVE THE SECOND STEP IN THE CASCADE OF CARE}

\begin{abstract}
${ }^{1,2}$ MG Van Veen, ${ }^{1} \mathrm{SCM}$ Trienekens, ${ }^{2} \mathrm{~T}$ Heijman, ${ }^{3} \mathrm{HM}$ Gotz, ${ }^{4} \mathrm{~S}$ Zaheri, ${ }^{1,5} \mathrm{G}$ Ladbury, ${ }^{6}$ D De Wit, ${ }^{2} \mathrm{JSA}$ Fennema, ${ }^{4} \mathrm{~F}$ De Wolf, ${ }^{1,7} \mathrm{MAB}$ Van Der Sande*. ${ }^{7}$ National Institute for Public Health and the Environment (RIVM), Centre for Infectious Disease Control, Bilthoven, The Netherlands; ${ }^{2}$ Public Health Service, Amsterdam, The Netherlands; ${ }^{3}$ Municipal Public Health Service Rotterdam-Rijnmond, The Netherlands; ${ }^{4}$ HIV Monitoring Foundation (SHM), Amsterdam, The Netherlands; ${ }^{5}$ EPIET, ECDC, Stockholm, Sweden; ${ }^{6}$ University of New South Wales, Centre for Social Research in Health; 'Julius Centre, University of Utrecht, The Netherlands
\end{abstract}

\subsection{6/sextrans-2015-052270.602}

Introduction The HIV cascade of care can identify missed opportunities to optimise control. The first step entails early testing, the second step is to ensure prompt linkage to care once diagnosed. To determine time to linkage to HIV-care following diagnosis at an STI centre, and to identify risk factors for delayed linkage.

Methods Patients newly diagnosed with HIV at STI clinics in the Netherlands were followed until linkage to care. Data were collected at time of diagnosis and at first consultation in care, including demographics, behavioural information, $\mathrm{CD}^{+}$counts and HIV viral load measurements. Delayed linkage to care was defined as $>4$ weeks between HIV diagnosis and first consultation.

Results 310 participants were included; the majority (90\%) men who have sex with men. For 259 participants (84\%) a date of first consultation in care was known; median time to linkage was 9 days (range 0-435). Overall, 95 (31\%) of participants were not linked within 4 weeks of diagnosis; among them, 44 were linked late and 51 were not linked at all by the end of study follow-up. Being young $(<25$ yrs $)$, having a non-Western ethnicity or lacking health insurance were independently associated with delayed linkage to care. Also, those being referred to care indirectly were more likely to have delayed linkage. Baseline $\mathrm{CD}^{+}{ }^{+}$count, viral load, perceived social support and stigma at diagnosis were not associated with delayed linkage. Risk behaviour and $\mathrm{CD}^{+}$counts declined between diagnosis and linkage to care.

Conclusions Although most newly diagnosed HIV patients were linked to care within 4 weeks, delay was observed for a third, with over half of them not yet linked at the end of follow-up. Vulnerable subpopulations (young, uninsured, ethnic minority) were at risk for delayed linkage. Testing those at risk is not sufficient, timely linkage to care needs to be assured.

Disclosure of interest statement This study was funded by the Netherlands Organisation for Health Research and Development (ZonMW) and the Ministry of Health, Welfare and Sport, the Netherlands. No pharmaceutical grants were received in the development of this study.

\section{P17.25 IMPACT ON COMPLIANCE WITH THE CHANGE OF FIRST LINE ANTI-RETROVIRAL DRUG REGIMENS AMONG PATIENTS ATTENDING ANTERETROVIRAL THERAPY CLINICS IN BLANTYRE, MALAWI}

Y Gadama*, S Sheikh, P Chasela. University of Malawi, College of Medicine, P/Bag 360, Blantyre, Malawi

\subsection{6/sextrans-2015-052270.603}

Introduction Change of any drug regimen impacts on compliance. Malawi changed the first line ARV regimen from stavudine-based (stavudine + lamivudine + nevarapine) to tenofovirbased regimen (tenofovir + lamivudine + efavirenz) because the former was associated with adverse side-effects and poor compliance. This study aimed at assessing the impact of the new ARV regimen on compliance.

Methods Using cross-sectional study, 169 participants recruited from 6 ART clinics randomly selected were interviewed to assess views on compliance, side-effects and satisfaction to new regimen. Self-reported data on compliance was complemented with patients' records at the clinics.

Results Compliance was poor in first visits on tenofovir-based regimen but gradually improved. Side-effects like dizziness, drowsiness and nightmares were reported in 56.9\%, 24.9\%, $23.1 \%$ of participants respectively especially during the first weeks and these negatively affected compliance (odds ratio = 1.5). Mean individual adherence was $93.3 \%$ on tenofovir-based and $85.5 \%$ on stavudine-based regimen ( $\mathrm{p}$ Value of $<0.0001$ ).

Conclusion Tenofovir-based regimen has improved long term compliance and has the potential to eliminate suboptimal compliance rates to ARVs being a drug taken once daily. However 